\title{
Design and Synthesis of Antifungal Compounds from 1,2,3-Triazoles through the Click Chemistry Approach
}

\author{
Ana Karla Estrada Valdés and Erick Cuevas-Yañez* \\ Centro Conjunto de Investigación en Química Sustentable UAEM-UNAM, Mexico
}

Submission: February 12, 2019; Published: March 12, 2019

"Corresponding author: Erick Cuevas Yañez Carretera Toluca-Atlacomulco Km 14.5, Toluca, Estado de México, 50200, Autonomous University of the State of Mexico, Mexico

\begin{abstract}
1,2,3-triazoles are easily prepared from CuAAC reaction and represent a potential source of antifungal compounds analogous to azole drugs. Accordingly, research groups have synthesized diverse 1,2,3-triazoles with modulated antifungal activity through the substituents in azide or alkyne precursors. A brief review of the state of the art about this topic is presented, focusing the increasing importance of developing new and more selective antifungal compounds.
\end{abstract}

Keywords: 1,2,3-triazole; Click Chemistry; Antifungal

Abbrevations: CuAAC: Copper (I) Catalyzed Azide Alkyne Cycloaddition

\section{Introduction}

Fungal infections directly affect million people each year. Besides the invasive fungal infections on humans, the plants and animals considered as a primary source of food are also susceptible to diseases caused by these eukaryotic microbes. This necessity for antifungals not only for medical purposes, but also for uses in agriculture and livestock farming, causes a high demand for this kind of compounds. A relevant commercially known group of antifungal agents are the azole drugs group, which is characterized by containing imidiazole or 1,2,4-triazole, an azole, in their structures. Discovered in the 1960s, some representative examples of azole antifungal drugs are miconazole or clotrimazole which been used as antifungal agents since the end of the 1970s [1,2]. Other examples are propiconazole, introduced in the market in 1979, fluconazole and ketoconazole (1990), itraconazole (1992), and second-generation azole drugs as voriconazole (2002) and posconazole (2006) [3].

A part of the success of azoles as antifungal drugs resides on the heterocyclic moiety which is related to the ability to inhibit $14 \mathrm{~s}$-demethylase at fungi cell membrane and subsequent ergosterol synthesis blocking by interaction with the cytochrome p450 enzyme complex. Hence, the presence of azole ring is fundamental to achieve a significant biological activity [4].

On the other hand, CuAAC reaction has been recognized as one of the most important reactions for molecular assembly which is concomitant with one of simplest and visionary chemical concepts developed in this century [5-7]. In this reaction, the formation of a 1,2,3-triazole inherent in this process has been used as linkage unit in the building of complex molecular structures, and more recently, in the drug design for diverse purposes. In this regard, excellent reviews give a general idea about this topic [8-12]. Due to the high degree of similarity between 1,2,3-triazole, imidazole and 1,2,4-triazole, an initial hypothesis is that 1,2,3-triazoles would display antifungal activity similar to commercially available azole drugs. The structures of these heterocyclic systems are represented in (Figure 1).

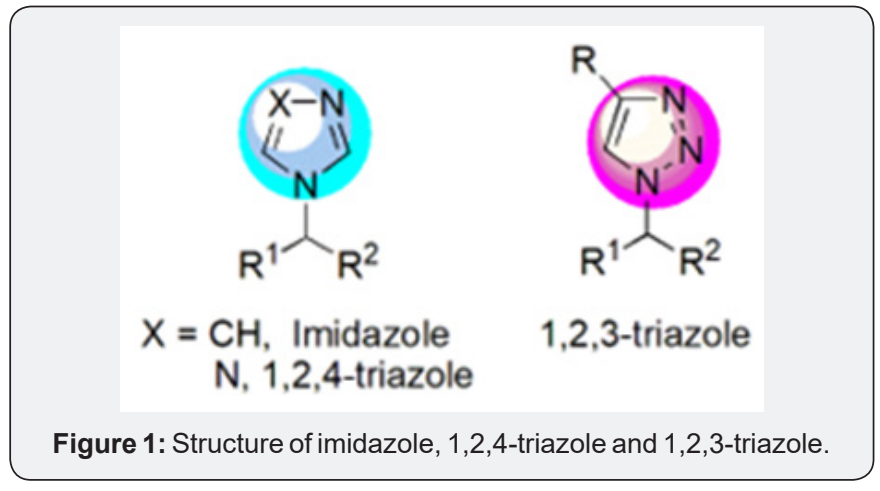

In fact, simple 1-benzyl-4-phenyl-1,2,3-triazole compound 1, figure 2 exhibits activity against M. tuberculosis H37Rv [13], 
as well as 1-benzyl-1,2,3-triazoles [2,14] and [3,15] resulted active against strains of Candida albicans. Other 1,2,3-triazoles (compounds 4 and 5, figure 2 bearing hydroxyl groups also showed antifungal activity compared to fluconazole reference standard [16,17]. Moreover, 1,2,3-triazole analogues to molecule 6 were available from CuAAC reaction of triclosan derivatives and displayed a significant activity against Candida albicans ATCC 10231 expressed in a high MIC50 and MIC90 [18,19].

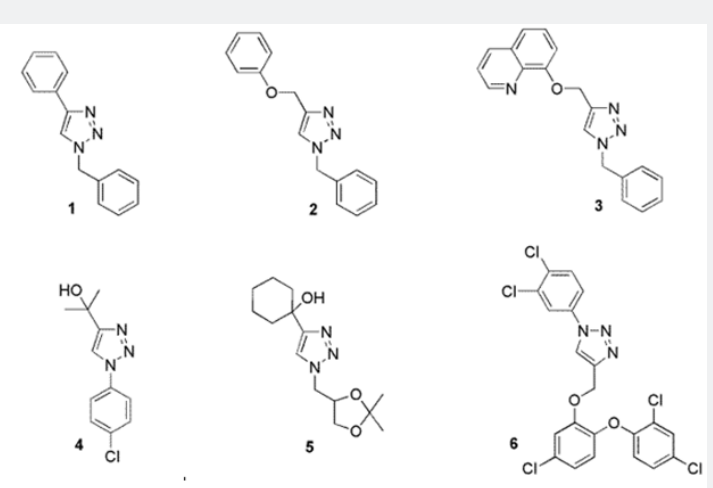

Figure 2: Structure of some benzyl and hydroxyl 1,2,3-triazoles.

Taking the advantages provided by CuAAC reaction and the Click Chemistry concept, some research groups have designed new 2-aryl-1-(1,2,4-triazolyl)-3-(1,2,3-triazolyl)propan2-ol derivatives as promising fluconazole analogues. Thus, 2-(2,4-Difluoro-phenyl)-1-[1,2,4]triazol-1-yl-pent-4-yn-2-ol (7) was reacted with diverse benzyl, alkyl azides and also azido bile acid derivatives to afford the corresponding triazoles 8 figure 3 [20-22]. An alternative strategy to increase the number of molecules in a chemical library from CuAAC reaction consists in changing the azide-alkyne group position on the reactive sites. With this idea, diverse alkynes were treated with 1-Azido-2-(2,4difluoro-phenyl)-3-[1,2,4]triazol-1-yl-propan-2-ol (9) yielding fluconazole analogues of type 10 [22-24]. All the compounds were highly active against different strains of fungi which prove that this kind of molecules possess an activity similar or higher than fluconazole having as further benefit that synthesis of these compounds is carried out with fewer reactions compared to the commercially available compound.

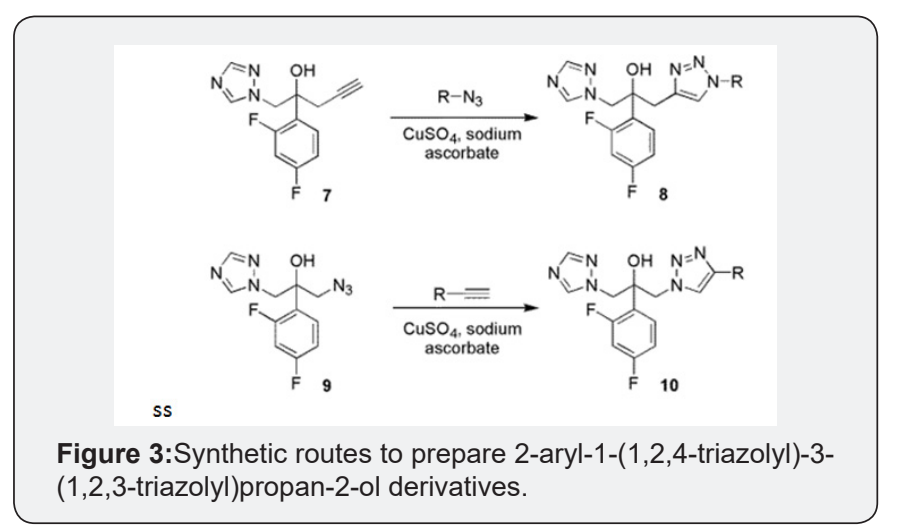

Figure 3 Synthetic routes to prepare 2-aryl-1-(1,2,4triazolyl)-3-(1,2,3-triazolyl) propan-2-ol derivatives

These examples demonstrate that 1,2,3-triazoles are useful structures for the design of antifungal compounds which offer a great chance to drive the research in this area taking in to consideration that the Click Chemistry will help to increase and modulate the biological properties through simplest reactions opening new trends in this area.

\section{References}

1. Lass-Flörl C (2011) Triazole Antifungal Agents in Invasive Fungal Infections: A Comparative Review. Drugs 71(18): 2405-2419.

2. Heeres J, Meerpoel L, Lewi P (2010) Conazoles. Molecules 15(6): 41294188.

3. Ngo HX, Garneau-Tsodikova S, Green KD (2016) A complex game of hide and seek: the search for new antifungals. Med ChemCommun 7(7): 1285-1306.

4. Sagatova AA, Keniya MV, Wilson RJ, Monk BC, Tyndall JDA (2015) Structural Insights into Binding of the Antifungal Drug Fluconazole to Saccharomyces cerevisiae Lanosterol 14 $\alpha$-Demethylase. Antimicrob Agents Chemother 59(8): 4982-4989.

5. Lahann, J (2009) Click Chemistry for Biotechnology and Materials Science, John Wiley \& Sons: Chichester, UK.

6. Chandrasekaran S (2016) Click Reactions in Organic Synthesis, WileyVCH Verlag: Weinheim, Germany.

7. Rutjes F, Fokin VV (2013) Click Chemistry, John Wiley \& Sons: Newyork, USA.

8. Bonandi E, Christodoulou MS, Fumagalli G, Perdicchia D, Rastelli G, et al. (2017) The 1,2,3-triazole ring as a bioisostere in medicinal chemistry. Drug Discov Today 22(10): 1572-1581.

9. Dheer D, Singh V, Shankar R (2017) Medicinal attributes of 1,2,3-triazoles: Current developments. Bioorg Chem 71: 30-54.

10. Haider S, Alam MS, Hamid H (2014) 1,2,3-Triazoles: scaffold with medicinal significance. Inflammation \& Cell Signaling 1: e95.

11. Thirumurugan P, Matosiuk D, Jozwiak K (2013) Click Chemistry for Drug Development and Diverse Chemical-Biology Applications. Chem Rev 113(7): 4905-4979.

12. Agalave SG, Maujan SR, Pore VS (2011) Click Chemistry: 1,2,3-Triazoles as Pharmacophores. Chem Asian J 6(10): 2696-2718.

13. Gallardo H, Conte G, Bryk F, Lourenço, Marilia S. Costa MS, Ferreira VF (2007) Synthesis and Evaluation of 1-Alkyl-4-phenyl-[1,2,3]-triazole Derivatives as Antimycobacterial Agent. J Braz Chem Soc 18: 12851291.

14. Aufort M, Herscovici J, Bouhours P, Moreau N, Girard C (2008) Synthesis and antibiotic activity of a small molecule's library of 1,2,3-triazole derivatives. Bioorg Med Chem Lett 18(3): 1195-1198.

15. Irfan M, Aneja B, Yadava U, Khan SI, Manzoor N, et al. (2015) Synthesis, QSAR and anticandidal evaluation of 1,2,3-triazoles derived from naturally bioactive scaffolds. Eur J Med Chem 93: 246-254.

16. Lima-Neto RG, Cavalcante NNM, Srivastava RM, Mendonça FJB, Almir G Wanderley AG et al. (2012) Synthesis of 1,2,3-Triazole Derivatives and in Vitro Antifungal Evaluation on Candida Strains. Molecules 17(5): 5882-5892.

17. Costa AV, Lacerda MV, Pinto RT, Moreira LC, Gomes EMC, et al. (2017) Synthesis of Novel Glycerol-Derived 1,2,3-Triazoles and Evaluation of 
Their Fungicide, Phytotoxic and Cytotoxic Activities. Molecules 22(10): 1666.

18. Layton TCF, Cuevas-Yañez E, Velasco-Montejo BE, Mendieta-Zerón H (2014) High susceptibility of Candida albicans ATCC 10231 to tetrahydrofuranosyl-1,2,3-triazoles obtained by click chemistry. Bolivian J Chem 31: 15-21.

19. Velasco BE, López-Téllez G, González-Rivas N, García-Orozco I, CuevasYañez E (2013) Catalytic activity of dithioic acid copper complexes in the alkyne-azide cycloaddition. Can J Chem 91: 292-299.

20. Zou Y, Zhao Q, Liao J, Hua H, Yu S, et al. (2012) New triazole derivatives as antifungal agents: Synthesis via click reaction, in vitro evaluation and molecular docking studies. Bioorg Med Chem Lett 22(8): 29592962.
21. Pore VS, Aher NG, Kumar M, Shukla PK (2006) Design and synthesis of fluconazole/bile acid conjugate using click reaction. Tetrahedron 62(48): 11178-11186.

22. Aher NG, Pore VS, Mishra NN, Kumar A, Shukla PK, et al. (2009) Synthesis and antifungal activity of 1,2,3-triazole containing fluconazole analogues. Bioorg Med Chem Lett 19(3): 759-763.

23. Wang S, Zhang L, Jin Y, Tang JH, Su H, et al. (2014) Synthesis and Evaluation of Some Substituted Heterocyclic Fluconazole Analogues as Antifungal Agents. Asian J Chem 26: 2362-2364.

24. Pore VS, Jagtap MA, Agalave SG, Pandey AK, Siddiqi MI, et al (2012) Synthesis and antifungal activity of 1,5-disubstituted-1,2,3-triazole containing fluconazole analogues. Med Chem Commun 3: 484-488.

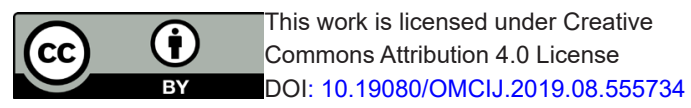

